\title{
The comparative and clinical study on Khandamalki and Patoladi kwatha in Amlapitta W.S.R to"Amlapittai Pryoktavya Kapha pittaha rovidhi”
}

\author{
Kumar Vipin ${ }^{1}$, Khatri Sapana ${ }^{2}$ and Meena Kedar Lal ${ }^{3}$ 约
}

Received: 24.09.2019

Revised: 12.10.2019

Accepted: 28.10.2019

\begin{abstract}
Amlapitta is one among the commonest disorders prevalent in the society nowadays due to indulgence in incompatible food habits and activities. It is a very common disease affecting mostly the adults. Acharya Vrinda in Vrind Madhavhas mentiond thatthe treatment of Amlapittamainly depends upon Kaphapittaharovidhi.So the present study was planned to prove this principle.Khandamalki and PatoladiKwatha, very simple, safe and cost effective drugs,have been chosen to explore the efficacyin the management of Amlapitta as Shaman therapy.shodhan therapy is time consuming and not possible to execute in all the patients so the present study has been carried out to evalute the Shamana effect of Khandamalki and PatoladiKwatha. The results of the study are discussed in this paper.
\end{abstract}

Key words: Amlapitta, Khandamalki, Patoladikwatha.

\section{Introduction}

Ayurveda is a holistic way of living in which the mind, body, diet and exercise act together to contribute to one`s health. Any vitiation leads to imbalance which needs to be corrected through regulation of diet, exercises, mind and bodily functions. In recent years, health levels are decreasing due to changing of life style, diet pattern, behavioral pattern and mental stress and strain. Everyone is prone to various disease due to the against of our normal physiology of digestion. There have been extraordinary increases disorders incidences related to Annavahastrotas (Gastrointestinal system) related disorders. Amlapitta is such type of G.I. disorder which is the outcome of faulty dietetic habits, with disturbed function of Agni (digestive fire) is the valuable topic of concern in the present era. Kashyapasamhita was the first text that gave a detailed description of Amlapitta (Kashyap Samhita). Kashyapasamhita has accepted the involvement of all three doshas in amlapitta whereas charakasamhita and madhavakara has Author's Address

${ }^{1}$ Department of Samhita, Sanskrit EvumSiddhanta, Uttrakhand Ayurved University, Gurukul Campus, Haridwar, Uttrakhand, India

${ }^{2}$ Dept of Ayurveda, State Govt. of Rajasthan, Rajasthan, India Department of Basic Principles, National Institute of Ayurveda, Jaipur, Rajasthan, India

E-mail.: dr.aroravipin@gmail.com accepted the dominance of pitta dosha in this disease. The word amlapitta is comprised of two words amla and pitta. The term amla refers to a particular type of taste equated with sour taste which causes excessive salivarysecretions. Pitta is a bodily chemical substance which is mainly responsible for the maintenance of the process of digestion and transformation. Amlapitta is a pathological condition in which there is vitiation of pitta dosha in the body pittadosha possesses katu rasa, but after it gets vitiated the katu rasa of pitta dosha changes to amla rasa (Kashyap Samhita).

Aims and objectives

The Comparative and Clinical Study on Khandamalki and PatoladiKwatha in AmlapittaW.S.R to "AmlapittaiPryoktavya Kaphapittaharovidhi" was undertaken with the following aims and objectives

1. Conceptual study on Amlapitta

2. To evaluate the efficacy of Khandamalki in the management of Amlapitta.

3. To evaluate the efficacy of PatoladiKwatha in the management of Amlapitta.

4. To evaluate the combined efficacy of Khandamalki and PatoladiKwatha in the management of Amlapitta. 
Materials and Methods

Following materials and methods were adopted for the completion of present research project:

Design of study: Simple, randomized, opens three group comparative studies.

Selection of cases: For clinical study, patients of Amlapittafulfilling the diagnostic criteria were registered from the OPD/IPD of NationalInstituteof Ayurveda, Jaipur (Agnivesha Charaka Samhita).

Diagnostic criteria were mainly based on the signs and symptoms of Amlapittadescribed in Ayurvedic classics. They include Avipaka, Klama, Utklesha, Tikt-Amlodgara, Hrita-Kanthadaha, AruchiGaurav, Chhardi, Shira-shul.

\section{(a) Inclusion Criteria}

i. Patient willing to undergo trial and ready to give written consent.

ii. Age : $16-60$ years

iii. Sex- either sex.

iv. Patients presenting with classical features of Amlapitta.

\section{(b) Exclusion Criteria}

i. Patients not willing for trial.

ii. Patients below the age of 16 years and above 60 years.

iii. Chronicity more than 5 years.

iv. Patients having organic disease like gastric ulcer, duodenal ulcer etc.

v. Patients suffering fromAmlapitta with any other chronic diseases like Asthma, Malignancies, Liver Cirrhosis, and Chronic renal failure, diabetes were excluded from the study.

\section{Research protocol}

\section{Administration of drugs}

For the present clinical study, 45 patients were enrolled and they were randomly divided into following three groups:-

GROUP A: 15 patients were registered in this group and they were given "Khandamalki" for 30 days.

GROUP B: 15 patients were registered in this group and they were given "PatoladiKwatha" for 30 days.

GROUP C: 15 patients were registered in this group and they were given "Khandamalki" and "PatoladiKwatha" for 30 days.
45 patients were selected for present study from Arogyashalaoutpatient department \& in patient department, National Institute of Ayurveda, Jaipur filling the inclusion criteria set for this purpose. Follow up was taken after 7 days. All patients were divided into three groups-

Group A- 15 clinically diagnosed patients of Amlapitta were administered "Khandamalki" 5 gram twice in a day with milk for 30 days.

Group B- 15 clinically diagnosed patients of Amlapitta were administered "Patoladikwatha" 20 $\mathrm{ml}$ twice in a day (before meal) with honey for 30 days.

Group C- 15 clinically diagnosed patients of Amlapitta were administered "Khandamalki" 5 gram twice in a day with milk for 30 days and "Patoladikwatha" $20 \mathrm{ml}$ twice in a day (before meal) with honey for 30 days.

\section{TRIAL DRUGS}

\section{GROUP A}

DRUG-khandamalki

Dose- 5 gram twice daily

Time of administration- after meal

Duration- 30 days

Anupana- milk

\section{GROUP B}

Drug-PatoladiKwatha

Dose- 20 gram twice daily

Time of administration- before meal

Duration- 30 days

Anupana- Honey

GROUP C

Drug -Khandamalki + PatoladiKwatha both

Pathological investigations

Routine hematological, urinary examinations were done before and after treatment to rule out any other pathology.

\section{Drugs}

\section{Khandamalki}

Pharmacodynamic properties of Khandamalki (B.R.

Shuladhikarshloka 228-32)

\section{PatoladiKwatha}

2. Pharmacodynamic properties of PatoladiKwatha (Amlapitta Nidana Chikitsa Prakrana Shloka 26) 
Table 1.

\begin{tabular}{|c|c|c|c|c|c|c|c|}
\hline $\mathbf{S N}$ & Drug & Botanical name & Rasa & Guna & Veerya & Vipaka & Karma \\
\hline 1 & Kushmanda & BeninkasaHispida & Madhura & Laghu, snigdha & Sheeta & Madhura & $\begin{array}{l}\text { Vata- pitta } \\
\text { shamak,tridoshahar }\end{array}$ \\
\hline 2 & Amalaki & Embilicaofficinalis & Pancharasa & Guru, ruksha & Sheeta & Madhura & Pitta shamak \\
\hline 3 & Pipalli & Piper longum & Katu & Laghu, snigdha & Anushnasheeta & Madhura & Kapha- vatashamak \\
\hline 4 & $\begin{array}{l}\text { Jiraka } \\
\text { (shweta) }\end{array}$ & Cuminamcyminum & Katu & Laghu, ruksha & Ushna & Katu & Kapha-vatashamak \\
\hline 5 & Shunti & Zingiberofficinale & Katu & Laghu, snigdha & Ushna & Madhura & Kapha- vatashamak \\
\hline 6 & Marich & Piprenigrum & Katu & Laghu, tikshna & Ushna & Katu & Kapha- Vatashamak \\
\hline 7 & Tallish- patra & Abieswebbiana & $\begin{array}{l}\text { Tikt- } \\
\text { madhura }\end{array}$ & Laghu- tikshan & Ushna & Katu & Kapha- Vatashamak \\
\hline 8 & Dhnyak & Coriandrumsativum & $\begin{array}{l}\text { Tikt- } \\
\text { madhura }\end{array}$ & Laghu- snigdha & Ushna & Madhura & $\begin{array}{l}\text { Pitta- Shamak } \\
\text { Tridoshhar }\end{array}$ \\
\hline 9 & Dal-chini & $\begin{array}{l}\text { Cinnamomnmzeylanicu } \\
\mathrm{m}\end{array}$ & Katu- tikt & Laghu, ruksha & ushna & Katu & $\begin{array}{l}\text { Kapha-Vatashamak, } \\
\text { Pitta Vardhak }\end{array}$ \\
\hline 10 & Tej-patra & Cinnamemmumtamala & Katu- tikt & Laghu, ruksha & Ushna & Katu & $\begin{array}{l}\text { Kapha- Vatashamak, } \\
\text { Pitta vardhak }\end{array}$ \\
\hline 11 & Suksha- ela & Elettariacardamomum & $\begin{array}{l}\text { Katu- } \\
\text { madhura }\end{array}$ & Laghu, ruksha & Sheeta & Madhura & Tridoshahar \\
\hline 12 & Nagkesar & Mesuaferrea & Tikt- kshya & Laghu, ruksha & Ushna & Katu & Kaphapittashamak \\
\hline 13 & Mustak & Cyperusrotundus & $\begin{array}{l}\text { Tikt, katu, } \\
\text { kshya }\end{array}$ & Laghu, ruksha & Sheeta & Katu & Kapha- pitta shamak \\
\hline 14 & Madhu & honey & madhura & Guru, ruksha & Sheeta & Katu & Kapha pitta shamak \\
\hline 15 & Ghrit & Ghee & madhura & Guru, snigdha & Sheeta & Madhura & Pitta shamak \\
\hline 16 & Khand & - & madhura & Snigdha, sheeta & Sheeta & Madhura & Pitta shamak \\
\hline
\end{tabular}

Table 2.

\begin{tabular}{|l|l|l|l|l|l|l|l|}
\hline SN & Drug & Botanical name & Rasa & guna & Veerya & Vipaka & Karma \\
\hline 1. & Patola & Trichosanthesdicoica & Tikta & Laghu, Ruksha & Katu & Ushna & Kaphapittashamak \\
\hline 2. & Amalaki & Emblicaofficinalis & Pancha rasa & Guru, ruksha & Sheeta & Madhura & Pitta shamak \\
\hline 3. & Haritaki & TermanaliaChebula & Pancharasa & Laghu, ruksha & Ushna & Madhura & $\begin{array}{l}\text { Tridoshharamainly } \\
\text { vatashamak }\end{array}$ \\
\hline 4. & Vibhitak & Termanaliabellirica & Kashya & Ruksha, laghu & Ushna & Madhura & Kaphapittashamak \\
\hline 5. & Nimba & AzadiractaIndica & Tikta, kashya & Laghu & sheeta & Katu & Kaphapittashamak \\
\hline 6. & Madhu & Honey & Madhura & Guru, ruksha & Sheeta & Katu & Kapha \\
& & & & & & & \\
\hline
\end{tabular}

\section{Criteria of assessment-}

Scoring system was adopted for assessment of various subjective features and grades from zero to four were accorded to various features according to the severity. The symptoms were evaluated and response of drug was recorded in term of percentage relief of symptoms. Patients were grouped under following categories on the basis of their results of the clinical trial.

- Completely relieved $-100 \%$ relief from symptoms

- Marked improvement-75-99\% relief from symptoms

- Moderate improvement-50-74\% relief from symptoms

- Mild improvement-25-50 \% relief from symptoms
- No improvement-below $25 \%$ or no relief

Statistical evaluation and result analysis

The entire data generated from clinical study was statistically analyzed. The results were made on the basis of grades of various variables compared between pre- trial and post- trial values in terms of percentage, based on mathematical means and its difference. Values between variables were compared with Wilcoxon Signed Ranks Test for dependent samples by using the degree of freedom $\mathrm{p}$ value. Intergroup comparison was also done with Kruskal-Wallis Test (Nonparametric ANOVA) and Dunn's Multiple Comparisons Test. The results were expressed in terms of mean, standard deviation (SD) and standard error (SE).

- $\quad \mathrm{P}<0.001$ - highly significant

- $P<0.01$ - significant

- $\quad$ P> 0.05 - non significant 
Results and Discussion

Table 1 Effect of therapy on assessment criteria in group A

\begin{tabular}{|c|c|c|c|c|c|c|c|c|c|c|}
\hline \multirow{2}{*}{ Symptoms } & \multirow{2}{*}{$\mathbf{N}^{1}$} & \multicolumn{3}{|c|}{ Mean } & \multirow{2}{*}{$\begin{array}{l}\text { Relief } \\
\%\end{array}$} & \multirow{2}{*}{$\begin{array}{l}\text { S.D. } \\
( \pm)\end{array}$} & \multirow{2}{*}{$\begin{array}{l}\text { S.E. } \\
( \pm)\end{array}$} & \multirow{2}{*}{$\mathbf{t}^{7}$} & \multirow{2}{*}{$\mathbf{P}^{8}$} & \multirow{2}{*}{ Results } \\
\hline & & BT $^{2}$ & $\mathbf{A T}^{3}$ & Diff. $^{4}$ & & & & & & \\
\hline Avipaka & 11 & 1.80 & 0.93 & 0.86 & 48.14 & 0.639 & 0.165 & 6.500 & .0005 & HS \\
\hline Klama & 11 & 1.46 & 1.20 & 0.26 & 18.18 & 0.457 & 0.118 & 2.256 & .0625 & NS \\
\hline Utklesha & 12 & 2.00 & 1.33 & 0.67 & 33.33 & 0.488 & 0.126 & 5.292 & .0020 & HS \\
\hline TiktAmlodgara & 15 & 2.53 & 1.60 & 0.93 & 36.84 & 0.798 & 0.206 & 4.525 & .0010 & HS \\
\hline Daha & 15 & 2.13 & 1.46 & 0.66 & 31.25 & 0.617 & 0.159 & 4.183 & .0020 & HS \\
\hline Chhardi & 10 & 1.40 & 0.86 & 0.53 & 38.09 & 0.743 & 0.191 & 2.779 & .0313 & S \\
\hline Shira-shul & 06 & 0.60 & 0.26 & 0.33 & 55.55 & 0.488 & 0.126 & 2.646 & .0625 & NS \\
\hline Gaurav & 06 & 0.53 & 0.26 & 0.26 & 50.00 & 0.457 & 0.118 & 2.256 & .0125 & $\mathrm{~S}$ \\
\hline Aruchi & 12 & 1.80 & 0.93 & 0.86 & 48.14 & 0.639 & 0.165 & 6.500 & .0005 & HS \\
\hline
\end{tabular}

$\left[{ }^{1}\right.$ No. of patients having symptoms, ${ }^{2}$ Mean score before treatment, ${ }^{3}$ Mean score after treatment, ${ }^{4}$ Difference in mean, ${ }^{5}$ Sandard deviation, ${ }^{6}$ Standard error, ${ }^{7}$ Paired t test value, ${ }^{8}$ Degree of freedom ]

$$
\text { Group- A }
$$

Fig 1.

\begin{tabular}{|c|c|c|c|c|c|c|c|c|c|c|}
\hline \multirow{2}{*}{ Symptoms } & \multirow{2}{*}{$\mathrm{N}^{1}$} & \multicolumn{3}{|c|}{ Mean } & \multirow{2}{*}{ Relief \% } & \multirow{2}{*}{$\begin{array}{l}\text { S.D. } \\
( \pm)\end{array}$} & \multirow{2}{*}{$\begin{array}{l}\text { S.E. } \\
( \pm)\end{array}$} & \multirow{2}{*}{$\mathrm{t}^{7}$} & \multirow{2}{*}{$\mathrm{P}^{8}$} & \multirow{2}{*}{ Results } \\
\hline & & $\mathrm{BT}^{2}$ & $\mathrm{AT}^{3}$ & Diff. $^{4}$ & & & & & & \\
\hline Avipaka & 12 & 1.73 & 0.73 & 1.00 & 57.69 & 0.654 & 0.169 & 5.916 & .0002 & HS \\
\hline Klama & 10 & 1.33 & 1.06 & 0.26 & 20.00 & 0.457 & 0.118 & 2.256 & .0625 & NS \\
\hline Utklesha & 15 & 2.33 & 1.53 & 0.80 & 34.28 & 0.560 & 0.144 & 5.527 & .0005 & HS \\
\hline TiktAmlodgara & 15 & 2.33 & 1.60 & 0.73 & 31.42 & 0.703 & 0.181 & 4.036 & .0039 & HS \\
\hline Daha & 15 & 1.86 & 1.00 & 0.86 & 46.42 & 0.351 & 0.090 & 9.539 & .0002 & $\mathrm{HS}$ \\
\hline Chhardi & 12 & 1.26 & 0.46 & 0.80 & 66.66 & 0.560 & 0.144 & 5.527 & .0010 & HS \\
\hline Shira-shul & 05 & 0.60 & 0.20 & 0.40 & 66.66 & 0.632 & 0.163 & 2.449 & .0625 & NS \\
\hline Gaurav & 07 & 0.93 & 0.46 & 0.46 & 50.00 & 0.516 & 0.133 & 3.500 & .0156 & $\mathrm{~S}$ \\
\hline Aruchi & 09 & 1.40 & 0.60 & 0.80 & 52.38 & 0.774 & 0.200 & 3.595 & .0039 & $\mathrm{HS}$ \\
\hline
\end{tabular}

Table 2 effect of therapy on assessment criteria in group $B$

${ }^{1}$ No. of patients having symptoms, ${ }^{2}$ Mean score before treatment, ${ }^{3}$ Mean score after treatment, ${ }^{4}$ Difference in mean,

${ }^{5}$ Sandard deviation, ${ }^{6}$ Standard error, ${ }^{7}$ Paired t test value, ${ }^{8}$ Degree of freedom

After analysis of above data of group A, it was significant result, whereas Klama and Shira-shul observed that the maximum relief in percentage show insignificant result (Agnivesha Charaka was in shira-shul (55.55\%), then in Gaurav Samhita).After analysis of above data of group B, it (50.00\%), then in Aruchi and Avipaka (48.14\%), was observed that the maximum relief in then in Chhardi (38.09\%), then in Tikt-amlodgara percentage was in Chhardi and Shira-shul $(36.84 \%)$, then in Daha $(31.25 \%)$ and minimum (66.66\%), then in Avipaka (57.69\%), then in relief in percentage was in Klama (18.18\%). Aruchi $(52.38 \%)$, then in Gaurav (50\%), then in According to statistical analysis, Avipaka, Utklesha, Daha (46.42\%), then in Utklesha (34.28\%), then in TiktAmlodgara,Daha, Aruchi shows highly Tikt-amlodgara (31.42\%) and minimum relief in significant result,Chhardi and Gaurav show percentage was in Klama (20\%). According to 10

Environment Conservation Journal 
statistical analysis, Avipaka, Utklesha, result, whereas Klama and Shira-shul show TiktAmlodgara, Daha, Chhardi and Aruchi shows insignificant result.

highly significant result, Gaurav show significant

Table 3. Effect of therapy on assessment criteria in group $\mathrm{C}$

\begin{tabular}{|c|c|c|c|c|c|c|c|c|c|c|}
\hline \multirow{3}{*}{ Symptoms } & \multirow{3}{*}{ N1 } & \multirow{2}{*}{\multicolumn{3}{|c|}{ Mean }} & \multirow{3}{*}{$\begin{array}{l}\text { Relief } \\
\%\end{array}$} & \multirow{3}{*}{$\begin{array}{l}\text { S.D.5 } \\
( \pm)\end{array}$} & \multirow{3}{*}{$\begin{array}{l}\text { S.E.6 } \\
( \pm)\end{array}$} & \multirow{3}{*}{ t7 } & \multirow{3}{*}{ P8 } & \multirow{3}{*}{ Results } \\
\hline & & & & & & & & & & \\
\hline & & BT $^{2}$ & $\mathbf{A T}^{3}$ & Diff $^{4}$. & & & & & & \\
\hline Avipaka & 14 & 2.46 & 0.73 & 1.73 & 70.27 & 0.961 & 0.248 & 6.985 & .0001 & HS \\
\hline Klama & 08 & 1.53 & 0.80 & 0.73 & 47.82 & 0.593 & 0.153 & 4.785 & .0020 & $\mathrm{HS}$ \\
\hline Utklesha & 13 & 2.20 & 1.20 & 1.00 & 45.45 & 0.645 & 0.169 & 5.916 & .0005 & HS \\
\hline TiktAmlodgara & 15 & 2.53 & 0.93 & 1.60 & 63.15 & 0.828 & 0.213 & 7.483 & .0002 & HS \\
\hline Daha & 15 & 2.33 & 0.93 & 1.40 & 60.00 & 0.632 & 0.163 & 8.573 & .0001 & HS \\
\hline Chhardi & 07 & 2.00 & 0.73 & 1.26 & 63.33 & 0.883 & 0.228 & 5.551 & .0010 & HS \\
\hline Shira-shul & 11 & 1.66 & 0.33 & 1.33 & 80.00 & 0.975 & 0.252 & 5.292 & .0010 & HS \\
\hline Gaurav & 09 & 1.46 & 0.40 & 1.06 & 72.72 & 0.961 & 0.248 & 4.298 & .0039 & HS \\
\hline Aruchi & 13 & 2.00 & 0.40 & 1.60 & 80.00 & 0.828 & 0.218 & 8.411 & .0001 & HS \\
\hline
\end{tabular}

${ }^{1}$ No. of patients having symptoms, ${ }^{2}$ Mean score before treatment, ${ }^{3}$ Mean score after treatment, ${ }^{4}$ Difference in mean,

${ }^{5}$ Sandard deviation, ${ }^{6}$ Standard error, ${ }^{7}$ Paired t test value, ${ }^{8}$ Degree of freedom

After analysis of above data of group C, it was (47.82\%) and minimum relief in percentage was in observed that the maximum relief in percentage Utklesha (45.45\%). According to statistical was inShira-shul and Aruchi (80\%), then in Gaurav analysis, Avipaka,klama, Utklesha, (72.72\%), then in Avipaka (70.27\%), then in TiktAmlodgara,daha, Chhardi, Shira-shul, Chhardi (63.33\%), then in Tikt-Amlodgara Gauravand Aruchi shows highly significant result. $(63.15 \%)$, then in Daha $(60 \%)$, then in Klama

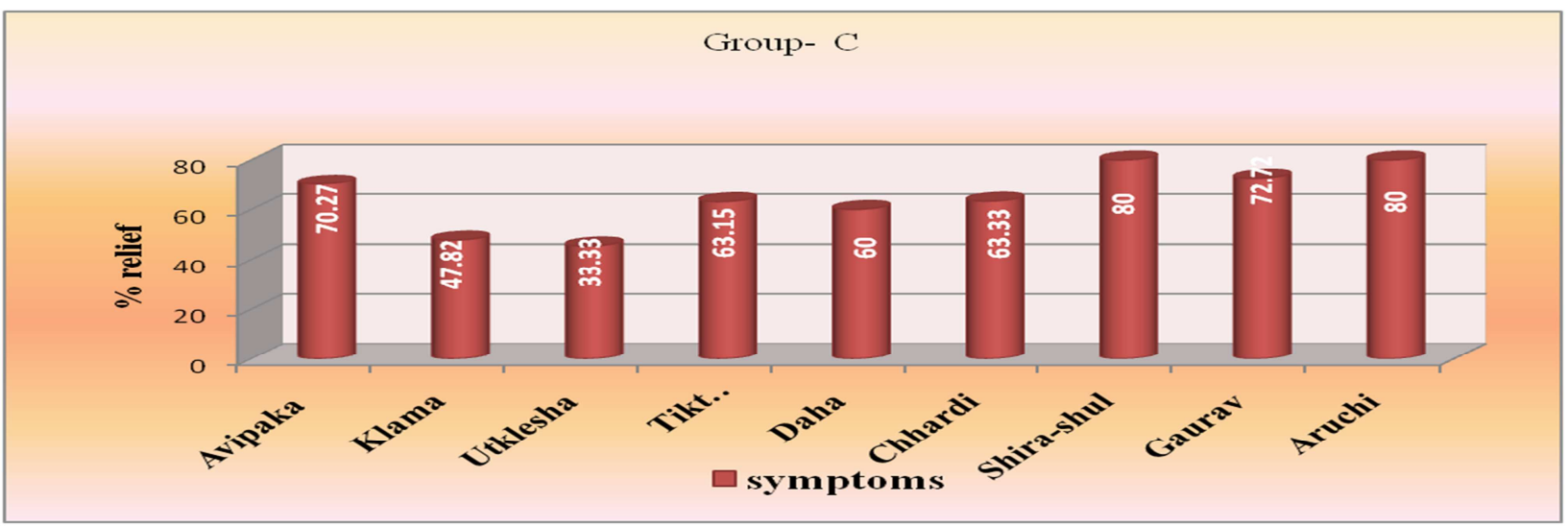

Fig 3. 
Kumar et al.

Table 4 - Intergroup comparison by Kruskal-Wallis Test (Nonparametric ANOVA)

\begin{tabular}{|l|l|l|l|l|}
\hline S.No. & Symptoms & KW Value & P Value & Results \\
\hline 1. & Avipaka & 7.582 & $\mathrm{P}<0.05$ & $\mathrm{~S}$ \\
\hline 2. & Klama & 7.013 & $\mathrm{P}<0.05$ & $\mathrm{~S}$ \\
\hline 3. & Utklesha & 6.132 & $\mathrm{P}<0.05$ & $\mathrm{~S}$ \\
\hline 4. & TiktAmlodgara & 9.107 & $\mathrm{P}<0.05$ & $\mathrm{~S}$ \\
\hline 5. & Daha & 11.092 & $\mathrm{P}<0.01$ & $\mathrm{HS}$ \\
\hline 6. & Chhardi & 6.339 & $\mathrm{P}<0.05$ & $\mathrm{~S}$ \\
\hline 7. & Shira-shul & 11.015 & $\mathrm{P}<0.01$ & $\mathrm{HS}$ \\
\hline 8. & Gaurav & 0.667 & $\mathrm{P}<0.05$ & $\mathrm{~S}$ \\
\hline 9. & Aruchi & 9.833 & $\mathrm{P}<0.01$ & $\mathrm{~S}$ \\
\hline
\end{tabular}

Table 5 Dunn's Multiple Comparisons Test

\begin{tabular}{|c|c|c|c|c|c|}
\hline S.No. & Symptoms & $\begin{array}{l}\text { Dunn's Multiple } \\
\text { Comparisons Test }\end{array}$ & $\begin{array}{l}\text { Mean Rank } \\
\text { Difference }\end{array}$ & P Value & Remarks \\
\hline \multirow{3}{*}{1.} & \multirow[t]{3}{*}{ Avipaka } & Group A \& Group B & -1.76 & $\mathrm{P}>0.05$ & NS \\
\hline & & Group A \& Group C & -11.63 & $\mathrm{P}<0.05$ & $\mathrm{~S}$ \\
\hline & & Group B\& Group C & -9.86 & $\mathrm{P}>0.05$ & NS \\
\hline \multirow{3}{*}{2.} & \multirow{3}{*}{ Klama } & Group A \& Group B & -0.00 & $\mathrm{P}>0.05$ & NS \\
\hline & & Group A \& Group C & -9.40 & $\mathrm{P}>0.05$ & NS \\
\hline & & Group B\& Group C & -9.40 & $\mathrm{P}>0.05$ & NS \\
\hline \multirow{3}{*}{3.} & \multirow[t]{3}{*}{ Utklesha } & Group A \& Group B & -2.33 & $\mathrm{P}>0.05$ & NS \\
\hline & & Group A \& Group C & -10.16 & $\mathrm{P}>0.05$ & NS \\
\hline & & Group B\& Group C & -7.83 & $\mathrm{P}>0.05$ & NS \\
\hline \multirow{3}{*}{4.} & \multirow{3}{*}{$\begin{array}{l}\text { TiktAmlodgar } \\
a\end{array}$} & Group A \& Group B & 2.53 & $\mathrm{P}>0.05$ & NS \\
\hline & & Group A \& Group C & -10.33 & $\mathrm{P}>0.05$ & $\mathrm{NS}$ \\
\hline & & Group B\& Group C & -12.86 & $\mathrm{P}<0.05$ & $\mathrm{~S}$ \\
\hline \multirow{3}{*}{5.} & \multirow[t]{3}{*}{ Daha } & Group A \& Group B & -3.73 & $\mathrm{P}>0.05$ & NS \\
\hline & & Group A \& Group C & -13.36 & $\mathrm{P}<0.01$ & $\mathrm{HS}$ \\
\hline & & Group B\& Group C & -9.63 & $\mathrm{P}>0.05$ & NS \\
\hline \multirow{3}{*}{6.} & \multirow[t]{3}{*}{ Chhardi } & Group A \& Group B & -4.73 & $\mathrm{P}>0.05$ & NS \\
\hline & & Group A \& Group C & -11.26 & $\mathrm{P}<0.05$ & $\mathrm{~S}$ \\
\hline & & Group B\& Group C & -6.53 & $\mathrm{P}>0.05$ & NS \\
\hline \multirow{3}{*}{7.} & \multirow[t]{3}{*}{ Shira-shul } & Group A \& Group B & -0.66 & $\mathrm{P}>0.05$ & NS \\
\hline & & Group A \& Group C & -12.83 & $\mathrm{P}<0.01$ & $\mathrm{HS}$ \\
\hline & & Group B\& Group C & -12.16 & $\mathrm{P}<0.05$ & S \\
\hline \multirow{3}{*}{8.} & \multirow[t]{3}{*}{ Gaurav } & Group A \& Group B & -3.80 & $\mathrm{P}>0.05$ & NS \\
\hline & & Group A \& Group C & -11.00 & $\mathrm{P}<0.05$ & S \\
\hline & & Group B\& Group C & -7.20 & $\mathrm{P}>0.05$ & NS \\
\hline \multirow{3}{*}{9.} & \multirow[t]{3}{*}{ Aruchi } & Group A \& Group B & 2.06 & $\mathrm{P}>0.05$ & NS \\
\hline & & Group A \& Group C & -11.06 & $\mathrm{P}<0.05$ & $\mathrm{~S}$ \\
\hline & & Group B\& Group C & -13.13 & $\mathrm{P}<0.05$ & S \\
\hline
\end{tabular}




\section{Overall assessment of therapy}

At the end of treatment each patient result were carefully observed to assess the overall effect of therapy

\begin{tabular}{|l|l|l|l|}
\hline Grading & Group A & Group B & Group C \\
\hline Complete relief & $0 \%$ & $0 \%$ & $0 \%$ \\
\hline Marked relief & $0 \%$ & $0 \%$ & $26.66 \%$ \\
\hline Moderate relief & $20 \%$ & $46.67 \%$ & $60.00 \%$ \\
\hline Mild relief & $53.33 \%$ & $53.33 \%$ & $6.67 \%$ \\
\hline No relief & $26.67 \%$ & $0 \%$ & $6.67 \%$ \\
\hline
\end{tabular}

In group A, $26.67 \%$ patients achieved no relief, $53.33 \%$ patients achieved mild relief, $20 \%$ patients achieved moderate relief, no any patients achieved marked and complete relief.

In group B, 53.33\% patients achieved mild relief, $46.67 \%$ patients achieved moderate relief, and no any patients achieved marked and complete relief.

In group C, $06.67 \%$ patients achieved no relief, $06.67 \%$ patients achieved mild relief, $60 \%$ patients achieved moderate relief, $26.66 \%$ patients achieved marked relief andno any patients achieved complete relief.

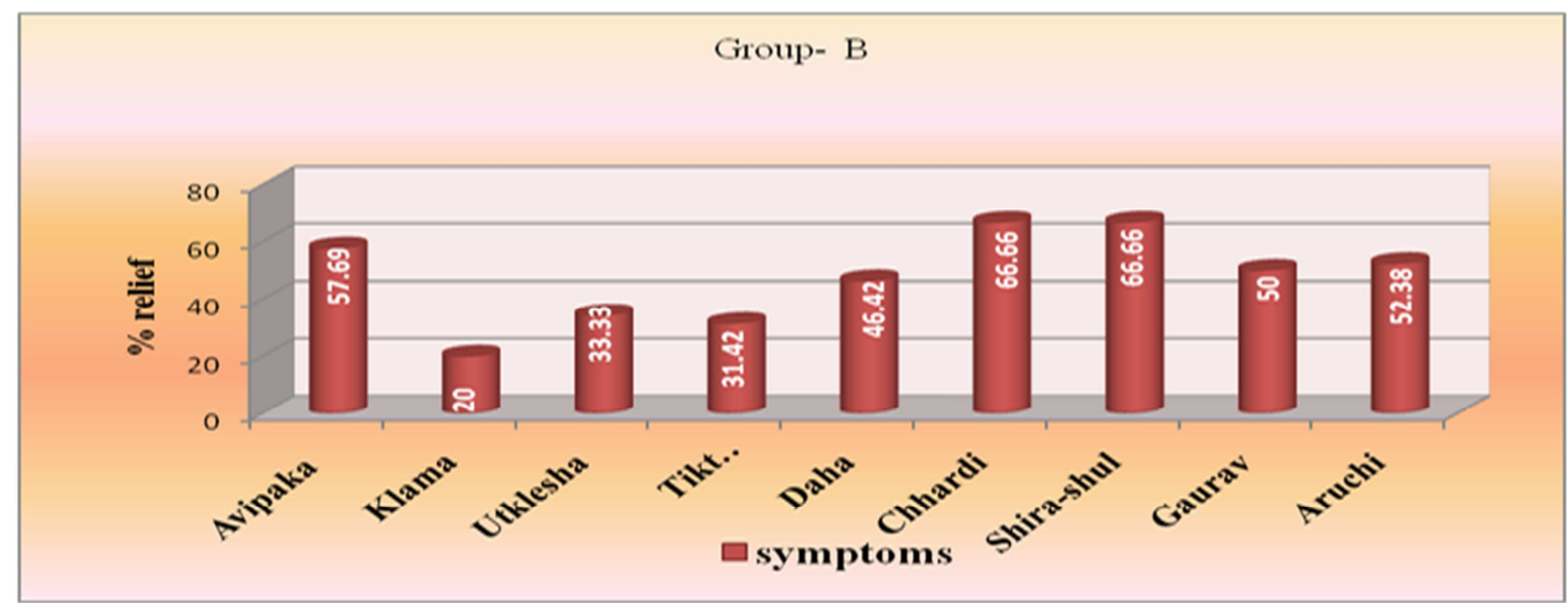

Fig 4.

Degradation by pathogenesis by formulated drugs-

Drug which is Agnideepaka, Kaphashamaka antagonist properties of VidagdhaPitta, Srotoshodhaka and Vatanulomaka is useful as palliative treatment of Amlapitta (Sushruta Sushruta Samhita). In Group AKhandamalaki with Anupana of luke warm milk was given. Main ingredients of Khandamalki, Kushmanda and Amalaki. The properties of Kushmanda are Laghu, Snigdha, SheetaVirya, MadhuraRasa and Vipaka. Due to its properties it suppresses Ushna and Tikshna properties of Vidagdhapitta. Amlaki is AmlaRasadominant Pancharasa, SheetaVeerya and MadhuraVipaka, Guru, Ruksha and Sheet. It is Pittashamak due to SeehtaVirya and MadhuraVipaka. Madhuis Pittakaphashamak due to Ruksha, Madhura and SheetVirya. Pippali, Jeeraka, Nagkeshar are Kaphavatshamak due to
Ruksha, Laghu, UshanaVirya and Katu, TiktaRasa. All of these drugs digest the SamAnsha of AmaRasa. There is a lot of importance of Anupana in increasing the activity of drug. There is Anupana of cow's milk which is also SheetaVirya andMadhuraRasa dominate and suppress the excess quantity of Pitta in Amlapitta. Mishriis also Pittashamak due to MadhuraRasa. Medhyaeffect of Kushmanda prevents its etiology due to psychological factor like Klam, Anidra etc.

PatoladiKwatha was given in group 'B' with Prakshep of Madhu. Ingredients of PatoladiKwatha are mostly Ruksha, Laghu,Tikt, Kasaya, AnushnaSheetaVeerya, MadhuraVipaka. Ruksha,Laghu properties and TiktaRasa dominant Dravya absorbs the Dravansha (liquid) of VidgdhaPachakaPitta. In formulated drug Kashaya rasa dominant Dravyas are Haritaka, Vibhitaki and Nimba. TiktaKashyaRasas are Kaphapittashamak. 


\section{Kumar et al.}

Triphalais mild laxative and Anulomna due to its Prabhava. Madhu as a PrakshepDravya, due to SheetaVirya, Ruksha, Kashaya and MaduraRasa is also Kaphapittashamak. Due to Sandhana property of Madhu it is also effective in complication of Amlapitta like gastric ulcer etc. by the analysis of ingredients of Patoladikwatha it is found that most of its ingredients are Kaphapittashamak which is just antagonistic of causative factors of Amlapitta and increasing the Agni. Due to increase in Agni proper digestion of Ahara will be possible and NiramaPitta will be produced not VidgdhaPitta. As a result of it AmalpittaVyadhi will not be produced.

\section{Conclusion}

Clinical study reveals that both drugsKhandamalki and PatoladiKwatha showed highly significant results. In group $\mathrm{C}$ the results were better than group A \& B. Because the combination of both drugs had been proved more effective in patients than single one. Hence, it can be concluded that the Amlapitta is better managed by administration of both drug without any side effect and "Kaphapittaharovidhi" is beneficial in the management ofAmlapitta disease.

\section{References}

Kashyap, KashyapSamhita by Vatsaya and VidyotiniHindi commentary by SatyapalaBhishagacharya, $3{ }^{\text {rd }}$ edition ChaukhambaSanskritSansthan Varanasi; KhilSthana, chapter 16, Shloka no. 1-49, p. 514-19

Agnivesha. CharakaSamhita,AyurvedaDipikaCommentary by Chakrapanidutta, revised edition ChaukhambhaSaurbharatiPrakashan, Varanasi ( 2005). ChikitsaSthana, chapter 15, Sholka no. 47, p. 517.

Agnivesha. CharakaSamhita, AyurvedaDipika commentary by Chakrapanidutta, revised edition ChaukhambhaSaurbharatiPrakashan, Varanasi 2005).SutraSthana, chapter 18, Sholka no. 50, p. 109.

Agnivesha. CharakaSamhita, AyurvedaDipikacommentary by Chakrapanidutta, revised edition ChaukhambhaSaurbharatiPrakashan, Varanasi ( 2005). ChikitsaSthana, chapter 15, Sholka no. 47, p. 517.

Sushruta: SushrutaSamhita with commentary of Dalhana, edited by VaidyaJadavajiTrikamajiAcharya, ChaukhambhaOrientalia, Varanasi, $8^{\text {th }}$ edition (2005)SutraSthana, Chapter 21, Shloka No. 11, p. 101.

Vrindmadhav: Vrindmadhav or Sidhyoga edited by Prof. PremvatiTiwari, ChaukhambhaVishwabharti, Varanasi, Chapter 53, Shloka no. 43 